\title{
Levantamento dos custos do diagnóstico e tratamento oncológico no paciente idoso
}

RESUMO | Objetivo: investigar o custeio do diagnóstico e tratamento de câncer em idosos de um Centro Oncológico de Minas Gerais. Método: Os dados foram coletados através de fonte secundária do Registro Hospitalar do Câncer, de um Hospital Filantrópico do Interior de Minas Gerais, entre o ano de 2009 a 2016, com uma amostra de 3.666 idosos em tratamento oncológico. A análise dos resultados foi feita por meio de estatística descritiva simples. Todos os aspectos éticos foram resguardados. Resultados: Entre as principais fontes de diagnóstico das neoplasias destaca-se a histologia do tumor, correspondendo a 85,50\%. Quanto ao custeio do diagnóstico e tratamento, em $25,09 \%$ e $86,05 \%$ dos casos, respectivamente, foram custeados pelo Sistema Único de Saúde. Conclusão: é importante ressaltar que o diagnóstico e tratamento precoce aumentam as chances de cura e que o Sistema Único de Saúde cobre a maioria dos custos, tornando possíveis as intervenções apropriadas para estes pacientes.

Palavras-chaves: Câncer; Idoso; Custos; Diagnóstico; Tratamento.

ABSTRACT | Objective: to investigate the cost of diagnosing and treating cancer in the elderly of an Oncology Center in Minas Gerais. Method: Data were collected through a secondary source of the Hospital Cancer Registry, from a Philanthropic Hospital in the Interior of Minas Gerais, between 2009 and 2016, with a sample of 3,666 elderly people undergoing cancer treatment. The results were analyzed using simple descriptive statistics. All ethical aspects were safeguarded. Results: Among the main sources of diagnosis of neoplasms, the histology of the tumor stands out, corresponding to $85.50 \%$. As for the cost of diagnosis and treatment, $25.09 \%$ and $86.05 \%$ of cases, respectively, were covered by the Unified Health System. Conclusion: it is important to note that early diagnosis and treatment increase the chances of cure and that Unified Health System covers most costs, making appropriate interventions for these patients possible.

Keywords: Neoplasms; Aged; Costs; Diagnosis; Therapy.

RESUMEN | Objetivo: investigar el costo del diagnóstico y tratamiento del cáncer en ancianos de un Centro de Oncología en Minas Gerais. Método: Los datos se recolectaron a través de una fuente secundaria del Registro Hospitalario de Cáncer, de un Hospital Filantrópico del Interior de Minas Gerais, entre 2009 y 2016, con una muestra de 3,666 ancianos en tratamiento oncológico. Los resultados se analizaron mediante estadística descriptiva simple. Se salvaguardaron todos los aspectos éticos. Resultados: Entre las principales fuentes de diagnóstico de neoplasias destaca la histología del tumor, correspondiente al 85,50\%. En cuanto al costo del diagnóstico y tratamiento, el 25,09\% y el $86,05 \%$ de los casos, respectivamente, fueron cubiertos por el Sistema Único de Salud. Conclusión: es importante señalar que el diagnóstico y el tratamiento precoces aumentan las posibilidades de curación y que Unified Health System cubre la mayoría de los costos, haciendo posibles las intervenciones adecuadas para estos pacientes. Palabras claves: Neoplasias; Ancioano; Costes; diagnóstico; Terapia.

\section{Juliana Costa Elias}

Enfermeira. Especialista em Oncologia. Enfermeira na Santa Casa de Alfenas.

ORCID: 0000-0002-0918-9810

\section{Andréa Cristina Alves}

Enfermeira. Doutora em Enfermagem. Docente do curso de enfermagem no IFSULDEMINAS, Passos-MG.

ORCID: 0000-0003-1535-4832

\footnotetext{
Aline Teixeira Silva

Enfermeira. Mestre em Enfermagem. Docente do curso de enfermagem da Universidade do Estado de Minas Gerais, Passos-MG.

ORCID: 0000-0001-9141-4775

\section{Monise Martins da Silva}

Enfermeira. Mestre em Enfermagem. Docente do curso de enfermagem da Universidade do Estado de Minas Gerais, Passos-MG. ORCID: 0000-0001-9141-4775
}

\section{Gabriela da Cunha Januário}

Enfermeira. Mestre em Enfermagem. Docente do curso de enfermagem da Universidade do Estado de Minas Gerais, Passos-MG.

ORCID: 0000-0002-6425-7433

Maria Inês Lemos Coelho Ribeiro
Enfermeira. Doutora em Enfermagem.
Docente do curso de enfermagem da
Universidade do Estado de Minas Gerais,
Passos-MG.
ORCID: 0000-0002-7684-2381

Recebido em: 06/09/2020

Aprovado em: 23/09/2020
INTRODUÇÃO

evando-se em consideração o aumento expressivo da população idosa, principalmente nos países em desenvolvimento, e as consequentes mudanças no perfil de doenças, com predominância das crônico-degenerativas $^{(1)}$. Estas doenças tem sido consideradas um problema de saúde pública, correspondendo a $80 \%$ dos óbitos e idosos nos países de baixa e média renda ${ }^{(2)}$.

Dentre elas podemos citar as cardiovasculares, doenças respiratórias crônicas e as neoplasias, sendo esta última responsável por uma das principais causas de mortalidade em todo o mundo, principalmente na população idosa ${ }^{(2)}$.

De acordo com a Organização Mundial da Saúde (OMS), é considerada ido- 
sa a pessoa que apresenta idade igual ou superior a 60 anos para países em desenvolvimento como o Brasil, e 65 anos em países desenvolvidos ${ }^{(3)}$.

O envelhecimento da população, bem como a mudança de perfil das doenças, aumenta a incidência de neoplasias malignas. Estima-se que para o ano de 2030 ocorram 26 milhões de casos novos de câncer diagnosticados e está doença ocorrerá em sua maioria nos idosos, população com maior risco de mortalidade devido suas vulnerabilidades ${ }^{(4)}$. Devido o crescimento da população e suas condições de saúde, houve uma motivação para o desenvolvimento de estudos para a melhor qualidade de vida desta população ${ }^{(5)}$.

As neoplasias estão crescendo cada vez mais em idosos, e o diagnóstico é um grande desafio, bem como o tipo de tratamento. O envelhecimento da população reflete muito ao diagnosticar o câncer e exige dos profissionais diferentes cuidados de saúde. O aumento deste tipo de doença fez com que o ocorresse um aumento de hospitalização destes idosos gerando, portanto, um aumento dos custos ${ }^{(6)}$.

O tratamento do paciente idoso com câncer deve ser realizado de uma forma qualificada, exigindo profissionais capacitados para o planejamento do tratamento. Os custos com estes idosos são altos, pois a maioria não possui apenas o câncer como patologia, podendo ter mais de uma doença de base, portanto o planejamento deve ser cauteloso, ponderando o risco e benefício do tratamento ${ }^{(4)}$.

Ressaltando que os custos gerados com cuidado do paciente idosos com câncer geram gastos finitos para atender as necessidades desta população, desde o diagnóstico que muitas vezes é tardio, a manutenção do tratamento até fase de cura ou terminal, e ainda o investimento em cuidados integrados, quando não há possibilidade de cura(7).

No Brasil o diagnóstico e tratamento do câncer são custeados pelo Sistema Único de Saúde (SUS), englobando em torno de $75 \%$, e pelos planos de saúde 25\%. Segundo Ministério da Saúde (MS), o custo com tratamento é bem mais extenso, devido ao não diagnóstico precoce destas neoplasias. A maioria dos custos estão relacionados a quimioterapia, seguida por cirurgia e radioterapia. As terapias por imunoterapias e terapias alvos ainda possuem valores altos e não são utilizadas em todos países, justamente por seu alto custo ${ }^{(8)}$.

Infelizmente a maioria dos diagnósticos é tardia, estimando que $60 \%$ dos casos já estão no estágio três e quatro, o que gera um aumento não só com tratamento, como hospitalização destes idosos. Nestes estágios, os custos são aproximadamente $60 \%$ a $80 \%$ maiores que os estágios um e dois, ressaltando a importância na prevenção das neoplasias ${ }^{(9)}$. Neste contexto o objetivo deste estudo foi investigar o custeio do diagnóstico e tratamento do câncer em pacientes idosos em um Centro Oncológico do Sul de Minas Gerais

\section{METODO}

Trata-se de um estudo descritivo, exploratório com abordagem quantitativa. A pesquisa foi realizada em um Hospital Filantrópico do Interior do Estado de Minas Gerais, por meio de dados secundários do Registro Hospitalar do Câncer (RHC) que é um sistema de armazenamento de informações com registro de pacientes com diagnóstico fechado de câncer, fornecendo dados de todos os pacientes atendidos pelos serviços de saúde, auxiliando na melhoria da assistência prestada ${ }^{(10)}$.

A amostra foi composta por idosos oncológicos cadastrados no RHC no período de 2009 a 2016 correspondendo a 3.666 cadastrados. A pesquisa foi realizada no ano de 2018. Não foram adicionados dados de 2017 ao estudo, pois até o momento não estavam disponíveis no sistema. A análise dos resultados foi feita por meio de estatística descritiva simples. Os dados obtidos foram apresentados por meio de tabelas e gráficos através do Programa Excel.

A pesquisa foi enviada para o responsável do Centro Oncológico e posteriormente autorizada. Em seguida o estudo foi submetido ao Comitê de Ética e Pesquisa da Instituição de Ensino, conforme o parecer 2.615.112 e número do CAAE 84183618.1.0000.8158. Justifica-se que, conforme o parecer do comitê de ética este estudo faz parte de um projeto maior com o título de "Caracterização da população idosa com câncer atendida em uma Unidade Oncológica do Sul de Minas Gerais".

\section{RESULTADOS}

Os resultados de presente estudo apontaram a caracterização das princi-

Tabela 1. Caracterização das principais bases para diagnóstico do câncer em idoso por meio do RHC de um hospital filantrópico do interior de Minas Gerais, no periodo de 2009 a 2016.

\begin{tabular}{|c|c|c|}
\hline Bases para diagnóstico & N & $\%$ \\
\hline Clínica & 231 & $6,50 \%$ \\
\hline Pesquisa clínica & 36 & $1,00 \%$ \\
\hline Exames por imagem & 58 & $1,50 \%$ \\
\hline Marcadores tumorais & 16 & $0,50 \%$ \\
\hline Citologia clínica & 174 & $4,74 \%$ \\
\hline Histologia metástase & 06 & $0,16 \%$ \\
\hline Histologia Tumor primário & 3141 & $85,50 \%$ \\
\hline Sem informação & 05 & $0,10 \%$ \\
\hline Total & 3666 & $100 \%$ \\
\hline
\end{tabular}

Fonte: Registro Hospitalar do Câncer, 2018. 
pais bases para diagnóstico de câncer, apresentando maior prevalência a histologia de tumor primário com $85,50 \%$, conforme apresentado na Tabela 1.

Quanto ao custeio do diagnostico realizado, o Gráfico 1 apresenta que em mais da metade dos casos prevaleceu a variável não se aplica, 61,19\%, seguida de diagnósticos custeados pelo Sistema Único de Saúde, 25,10\%. Importante ressaltar que no RHC não especifica ou descreve o que seriam as variáveis sem informação ou outros.
Em relação ao custo do tratamento realizado, em 86,06\% dos casos foram realizados pelo SUS, seguido da variável não se aplica, 13,03\%. O Gráfico 2 apresenta esta distribuição.

\section{DISCUSSÃO}

Nos últimos anos o Brasil tem realizado esforços para a expansão da assistência oncológica à população, aumentando consideravelmente a quantidade de procedimentos oncológicos realiza-
Gráfico 1. Distribuição do custo de diagnóstico do tumor por operadoras e pelo

Sistema Único de Saúde por meio do RHC de um hospital filantrópico do interior de Minas Gerais, no período de 2009 a 2016.

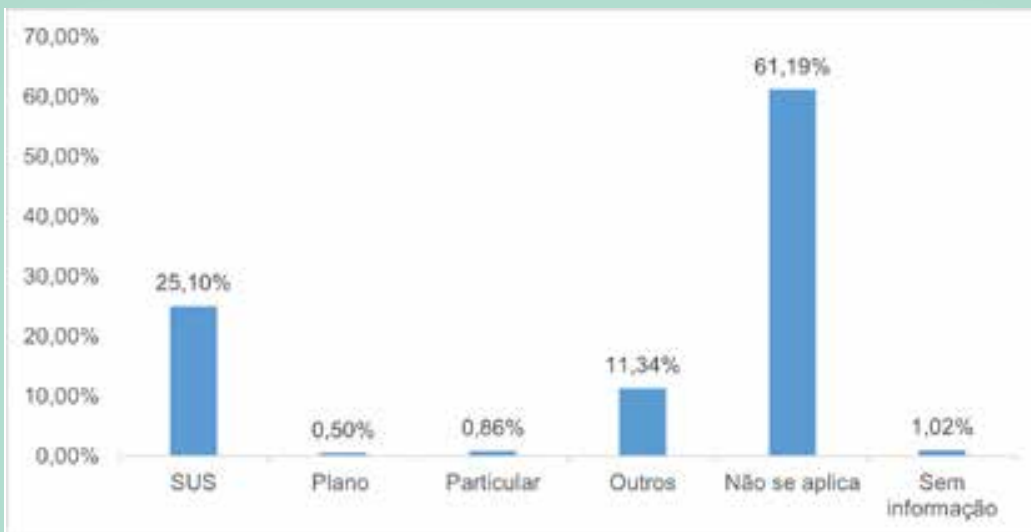

Fonte: Registro Hospitalar do Câncer, 2018.

Gráfico 2. Distribuição do custo do tratamento do tumor por operadoras e pelo Sistema único de saúde por meio do RHC de um Hospital Filantrópico do Interior de Minas Gerais, no período de 2009 a 2016.

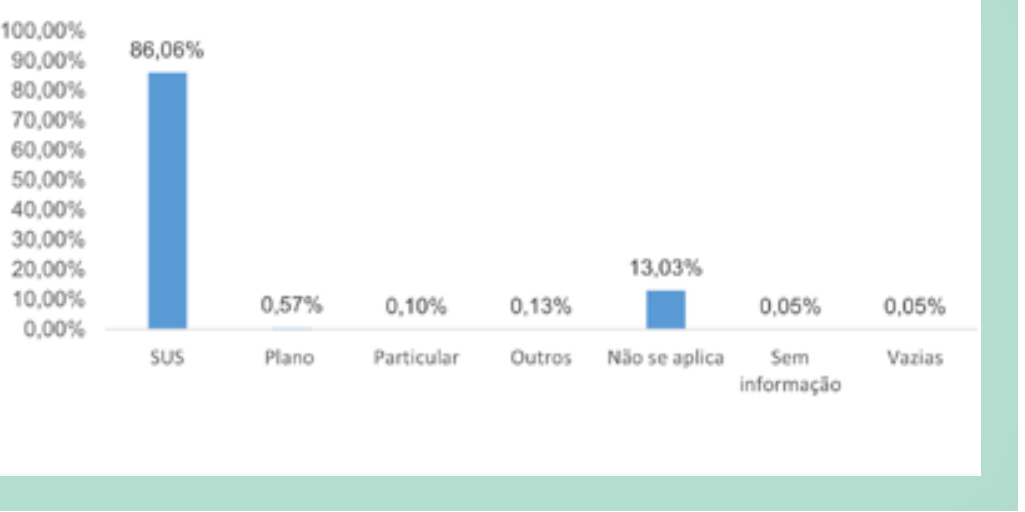

Fonte: Registro Hospitalar do Câncer, 2018. dos no país e o número de instituições de saúde do SUS voltadas para atendimento ao câncer. O aumento nos estabelecimentos de saúde, nos últimos 15 anos, expandiu em aproximadamente $71,3 \%$. Esse crescimento não se mostrou homogêneo em todas as regiões, variando de $333 \%$ a $50 \%$, na região norte e centro-oeste, respectivamente ${ }^{(11)}$.

As normativas do SUS, por meio da Política Nacional de Atenção Oncológica (PNAO) de 2005 institui a integralidade da assistência à pessoa com câncer, o que inclui ações desde a promoção e prevenção até o diagnóstico, tratamento, reabilitação e cuidados paliativos ${ }^{(12-13)}$. O diagnóstico precoce ainda é a meIhor estratégia para o planejamento das ações e tratamento dos pacientes oncológicos. Segundo o $\mathrm{MS}^{(14)}$, para cada tipo de tumor há um exame específico, destacando-se o exame clínico, por imagem e a histologia do tumor.

Corroborando com os achados deste estudo, uma pesquisa realizada ${ }^{(15)}$ demonstrou que a histologia do tumor primário é a principal base para diagnóstico do câncer, o que pode auxiliar também no planejamento da terapêutica.

Quanto ao financiamento do diagnóstico oncológico, nesta pesquisa mais da metade foi relacionado à variável não se aplica, seguido pelo SUS. Importante ressaltar que no RHC não especifica o significado desta variável. Um estudo encontrado na literatura relatou que a maior dificuldade do financiamento é garantir a universalidade e integralidade do paciente idoso com câncer, principalmente devido às desigualdades sociais e regionais existentes no Brasil( ${ }^{(9)}$.

$\mathrm{Na}$ presente pesquisa, mais da metade dos tratamentos realizados em pacientes oncológicos, são custeados pelo SUS. Estes resultados corroboram com outra pesquisa encontrada na literatura, que demostrou que os tratamentos são custeados pelos SUS desde as medicações até a internação $o^{(7)}$.

O tratamento do câncer é considerado de alto custo devido aos inúmeros ti- 
pos e dos protocolos instituídos para cada tipo de neoplasia, bem como os gastos com quimioterapias, cirurgias, hormonioterapia, imunoterapias e cuidados integrados a estes pacientes. As instituições devem ser cadastradas e o repasse acontece por ciclos de atendimento ${ }^{(16)}$, cabendo aos gestores de saúde a administração dos recursos recebidos ${ }^{(17)}$. Ressalto que o RHC não cita os tipos de tratamentos, apenas o primeiro tratamento realizado nestes idosos.

Neste contexto, a literatura sugere que os custos com o tratamento de pacientes oncológicos são maiores no primeiro ano após diagnóstico e na fase final da doença, devendo- se a isso maior número de internações na fase terminal ${ }^{(18)}$. Após um ano de diagnóstico, os custos tendem a se estabilizar, diminuindo significativamente com o estadiamento e extensão da neoplasia, devendo os serviços de saúde estar preparados para atender as demandas iniciais destes pacientes ${ }^{(7)}$.

\section{CONCLUSÃO}

Os custos com diagnóstico e tratamento do câncer ainda são considerados caros, pois se utiliza de vários instrumentos e protocolos para que seja realizado da melhor forma possível. Este estudo possibilitou identificar as principais bases de diagnóstico do câncer e seus custos. É importante ressaltar que o SUS financia tanto o diagnóstico quanto o tratamento dos pacientes idosos com câncer em qualquer estágio da doença.

Importante ressaltar que o diagnóstico precoce é a melhor forma de cura e que se necessário maiores investimentos em programas de prevenção e ações no combate ao câncer, favorecendo assim o sucesso no tratamento e a melhor qualidade de vida desta população. Espera-se que este estudo possa contribuir para o avanço e registro do conhecimento nesta área de grande relevância social, com enfoque específico nos custos, constituindo assim, referência para outros trabalhos e aprofundamentos.

\section{Referências}

1. Júnior $B S$, Oliveira $L P A B$, Silva RAZ. Doenças crônicos não transmissíveis e a capacidade funcional de idosos. Journal of Research Fundamental Care Online, 2014, 6(2): 516-52. Disponível em: https://www.redalyc.org/ $\mathrm{html} / 5057 / 505750622008 /$. Acesso em 19 dez. 2018.

2. Carvalho MHR, et al. Tendência de mortalidade de idosos por doenças crônicas no município de Marília-SP, Brasil: 1998 a 2000 e 2005 a 2007. Epidemiol. Serv. Saúde, 2014, 23(2); 347-354. Disponível em: https://www. scielosp.org/pdf/ress/2014.v23n2/347-354/pt. Acesso em 21 mar. 2020. 3. Brasil. Organização Mundial da Saúde: Relatório Mundial de envelhecimento e saúde. 2015. Disponível em: https://sbgg.org.br/wp-content/uploads/2015/10/OMS-ENVELHECIMENTO-2015-port.pdf. Acesso em 19 mar. 2020.

4. Ferreira MLL, et.al. Qualidade de vida relacionada a saúde de idosos em tratamento quimioterápico. Revista Brasileira de Geriatria e Gerontologia, 2015, 18(1); 167-177. Disponível em: https://www.redalyc.org/ pdf/4038/403839881015.pdf. Acesso em 19 mai. 2020.

5. Pimenta $F B$, et.al. Fatores associados a doenças crônicas em idosos atendidos pela Estratégia de Saúde da Família. Ciência e Saúde Coletiva, 2015, 20(8). Disponível em: https://www.scielosp.org/scielo.php?pi$\mathrm{d}=\mathrm{S} 1413-81232015000802489 \&$ script=sci_arttext. Acesso em 21 abr. 2020. 6. Formiga MYG. Hospitalizações por neoplasias em idosos no âmbito do sistema único de saúde na Paraíba/Brasil. Revista Saúde e Pesquisa, 2015, 8(3); 479-491. Disponível em: http://docs.bvsalud.org/biblioref/2017/02/831975/08_rilva_revisado_ing.pdf. Acesso em 01 jan. 2020.

7. Knust RE, et.al. Estimativa dos custos da assistência do câncer em um hospital público de referência. Revista de saúde pública. 2017. Disponível em: http://www.scielo.br/pdf/rsp/v51/pt_0034-8910-rsp-S1518-87872017051006665.pdf. Acesso em 06 jan. 20120.

8. Floresti F. Câncer: apesar do avanço de tratamentos, custos ainda são empecilhos. Revista Galileu. 2018. Disponível em: https://revistagalileu.globo. com/Revista/noticia/2018/03/cancer-apesar-do-avanco-de-tratamentos-custos-ainda-sao-empecilhos.html. Acesso em 16 de abr. 2019.

9. Knust RE. Estimativas dos custos diretos da assistência oncológica do câncer de pulmão não pequenas células avançadas em um hospital público de referência. 2015. Dissertação (Título de mestre em ciências de Saúde Pública) - Fundação Oswaldo Cruz. Rio de Janeiro. Disponível em: https:// bvssp.icict.fiocruz.br/lildbi/docsonline/get.php?id=4281. Acesso em 23 de abr. 2020.
10. Brasil. Instituto Nacional de Câncer José de Alencar Gomes da Silva (INCA) / Ministério da Saúde. Registros Hospitalares de Câncer: planejamento e gestão. 2010. Disponivel em: https://www.inca.gov.br/sites/ufu.sti.inca. local/files//media/document//registros-hospitalares-de-cancer-2010.pdf Acesso em 18 de maio de 2019.

11. Migowski A, et al. A Atenção Oncológica e os 30 Anos do Sistema Único de Saúde. Revista Brasileira de Cancerologia, 2018; 64(2): 247-250. Disponível em: https://rbc.inca.gov.br/site/arquivos/n_64/v02/pdf/14-artigo-de-opiniao-a-atencao-oncologica-e-os-30-anos-do-sistema-unico-de-saude.pdf. Acesso em: 22 set 2020.

12. Gadelha MIP. Planejamento da assistência oncológica: um exercício de estimativas. Rev Bras Cancerol. 2002;48(4):533-43.

13. Renna Junior NL. Acesso a diagnóstico e tratamento do câncer de mama e colo uterino no Brasil: analise dos dados dos registros hospitalares de câncer [dissertação]. Rio de Janeiro: Universidade do Estado do Rio de Janeiro; 2016.

14. Brasil. Organização Mundial da Saúde: Relatório Mundial de envelhecimento e saúde. 2015. Disponível em: https://sbgg.org.br/wp-content/uploads/2015/10/OMS-ENVELHECIMENTO-2015-port.pdf. Acesso em 19 Dez. 2018

15. Debiase $M$, et, al. Perfil epidemiológico e análise de sobrevida de pacientes com câncer de pulmão a partir da primeira consulta em um centro terciário de oncologia/SUS. Revista Brasileira de Oncologia Clínica, 2010, 7(22). Disponível em: https://www.sboc.org.br/sboc-site/revista-sboc/pdfs/22/artigo16.pdf. Acesso em 16 de maio 2018.

16. Brasil. Instituto Nacional de Câncer José de Alencar Gomes da Silva (INCA) / Ministério da Saúde. Registros Hospitalares de Câncer. 2016. Disponível em: http://www.inca.gov.br/conteudo_view.asp?id=351. Acesso em 16 de nov. 2017.

17. Kos SR, et.al. Repasse do SUS vs custo dos procedimentos hospitalares: É possível cobrir os custos com o repasse do SUS? XXII Congresso Brasileiro de Custos - Foz do Iguaçu, PR, Brasil, 11 a 13 de novembro de 2015. Anais... Foz do Iguaçu: UFPR, 2015, p.1-16.Disponível em: https://anaiscbc.emnuvens.com.br/anais/article/viewFile/4026/4027. Acesso em 05 de maio 2019. 18. Cipriano $L E$, et al. Lung cancer treatment costs, including patient responsibility, by disease stage and treatment modality, 1992 to 2003. Value Health. 2011;14(1):41-52. Disponivel em: https://pubmed.ncbi.nlm.nih. gov/21211485/. Acesso em: 23 set. 2020. 